\title{
Stress Effects on Defects and Dopant Diffusion in Si
}

\section{Citation}

Aziz, Michael J. 2001. Stress effects on defects and dopant diffusion in Si. Materials Science in Semiconductor Processing 4, (5): 397-403.

\section{Published Version}

http://dx.doi.org/10.1016/S1369-8001(01)00014-2

\section{Permanent link}

http://nrs.harvard.edu/urn-3:HUL.InstRepos:2797446

\section{Terms of Use}

This article was downloaded from Harvard University's DASH repository, and is made available under the terms and conditions applicable to Other Posted Material, as set forth at http:// nrs.harvard.edu/urn-3:HUL.InstRepos:dash.current.terms-of-use\#LAA

\section{Share Your Story}

The Harvard community has made this article openly available.

Please share how this access benefits you. Submit a story.

Accessibility 
Invited review for Materials Science in Semiconductor Processing

\title{
Stress Effects on Defects and Dopant Diffusion in Si
}

\author{
Michael J. Aziz ${ }^{a)}$ \\ Division of Engineering and Applied Sciences, Harvard University, Cambridge, MA 02138
}

\begin{abstract}
The effects of stress on equilibrium point defect populations and on dopant diffusion for single crystals free of extended defects are reviewed. The thermodynamic relationships presented permit the direct comparison of hydrostatic and biaxial stress experiments and of atomistic calculations under hydrostatic and nonhydrostatic stress for any proposed mechanism. Experiments on the effects of pressure and stress on the diffusivity are reviewed. For $\mathrm{Sb}$ in $\mathrm{Si}$, using as input the measured effect of hydrostatic pressure on diffusion and the assumption of a pure vacancy mechanism, the measured effect of biaxial stress on diffusion can be predicted successfully with no free parameters. For B in $\mathrm{Si}$, an apparent discrepancy between the hydrostatic and biaxial stress effects exists assuming a pure interstitial-based mechanism. The requirements to permit the prediction of the effect of an arbitrary stress state on diffusion in an arbitrary direction are discussed.
\end{abstract}

\section{INTRODUCTION}

Because understanding and controlling diffusion-related phenomena become increasingly important as semiconductor device dimensions decrease, diffusion in Si has been heavily studied [1]. For bandgap engineering purposes, biaxial strain is designed into certain epitaxial semiconductor devices, e.g. heterojunction bipolar transistors for high-power and high-speed applications such as wireless communications. The study of stress effects on diffusion, which have only recently been characterized experimentally [2-8], is an important part of the study of the stability of such strainedlayer epitaxial materials. Additionally, although bulk wafers cannot sustain significant nonhydrostatic stresses at diffusion temperatures, such stresses are sustained near interfaces with patterned films and in the films themselves. These stresses in integrated circuit materials and other multi-layer devices can 
be quite large due to growth stresses, interfacial stresses, thermal expansion mismatch, or dislocations [9]. The complexities associated with nonhydrostatic stress states in these materials (as well as in initially biaxially strained materials after the breakdown of a smooth, flat film morphology) make the interpretation of stress effects in terms of basic mechanisms and the prediction of stress effects from known mechanisms quite difficult. However, in certain cases, hydrostatic pressure and simple nonhydrostatic stress states can provide sufficient information to permit the prediction of behavior under arbitrary stress states [10-13].

The atomic diffusivity in a material with cubic crystallography is given by

$$
\begin{aligned}
& D=\frac{1}{6} \lambda^{2} \vee f \exp \left(-\frac{G^{*}}{k_{\mathrm{B}} T}\right) ; \\
& G^{*}=G_{+} G^{m},
\end{aligned}
$$

where $\lambda$ is the jump distance, $v$ is an effective vibrational frequency [14], $G^{*}$ is the Gibbs free energy of activation, $G f$ is the Gibbs free energy of formation of the mobile species, $G^{m}$ is the Gibbs free energy of migration of the mobile species, and departures from a true random-walk are accounted for by the correlation factor $f$. For example, for diffusion of an impurity "A" by a normal vacancy mechanism, the mobile species (which is called an A-V complex) is A on a lattice site bound to a vacancy, and $G f$ is the change in Gibbs free energy when one vacancy is created at a kink site on the surface and placed next to the impurity. The free energy of migration $G^{m}$ is the change in free energy when the mobile species moves to the saddle point of its migration path.

\section{DIFFUSION UNDER STRESS}

To understand the various influences of pressure and stress on the diffusivity [10,11], one must consider both the effects on the point defect concentrations $[9,10,15,16]$ and on the point defect mobilities [17,18].

The thermodynamics of point defect formation in heavily dislocated crystals under hydrostatic or nonhydrostatic stress has been well understood for decades. When experimental conditions are such that the point defect concentrations equilibrate with sources and sinks rapidly compared to the 
experimental time scale, then the pressure-dependence of the point defect concentration is characterized by the point defect formation volume $V f$, a scalar. The new situation that gives the point defect formation volume in a cubic crystal a tensor character is that large single crystals entirely free of extended defects, at which point defects can internally equilibrate, are now commonplace.

Consequently, in a single crystal free of internal point defect sources, the volume change upon point defect formation does not tell the entire story: the crystal changes shape, the shape change depends upon which surface(s) are sources/sinks for defect equilibration ${ }^{\dagger}$, and stresses interact with the components of that shape change in the characteristic stress • strain tensor manner.

Let us focus on the diffusion coefficient in the direction normal to an (001) surface, which is the direction measured in practically all experiments, and let the dopant diffusivity in this direction be $D_{33}(T, \sigma)$, where $\sigma$ is the stress tensor. The effect of stress on $D_{33}$ is characterized by the activation strain tensor $[10,11] \mathbf{V}^{*}$ :

$$
V_{\mathrm{ij}}^{*}=k_{\mathrm{B}} T\left(\frac{\partial \ln D_{33}}{\partial \sigma_{\mathrm{ij}}}\right) \text {. }
$$

Under hydrostatic pressure $\sigma_{11}=\sigma_{22}=\sigma_{33}=-p$ and the pressure effect is therefore minus the sum of these three uniaxial effects. Thus the pressure effect is characterized by scalar activation volume $V^{*}$ :

$$
V^{*}=-k_{\mathrm{B}} T\left(\frac{\partial \ln D}{\partial p}\right)
$$

$V^{*}$ is therefore the sum of the three diagonal elements of the activation strain tensor* . The activation

$\dagger$ For example, the thermal expansion coefficient of a thin wafer of a material with cubic crystallography that is free of extended defects is greater in the thin direction than in the orthogonal directions because most of the thermally injected point defects come from the thin direction.

* In fact, to encompass cases in which several mechanisms contribute significantly to transport, (3) and (4) are more properly interpreted as the apparent activation strain tensor and volume arising 
volume can also be written as the pressure-derivative of the Gibbs free energy of activation:

$$
V^{*}=\left(\frac{\partial G^{*}}{\partial p}\right) .
$$

We can interpret (5) to mean that pressure affects the diffusivity through a Boltzmann factor in the change in $G^{*}$ in Eq. (1), where the change is due to the $p V$ work done by the pressure medium against the volume changes associated with defect formation and migration $\%$. These changes are shown schematically in Fig. 1.

Under nonhydrostatic stress, the Gibbs free energy is difficult to define, but the stress-induced change in diffusivity can still be written as a Boltzmann factor in the work done by the interaction of the activation strain tensor and the stress state [18] as indicated below. As the activation strain tensor is the sum of the formation strain tensor and the migration strain tensor, let us consider them separately.

For point defects formed at the (001) surface, which is what we expect for diffusion measured near the free surface of any dislocation-free (001) wafer or thin film, the dimension changes of the crystal upon point defect formation can be described by the formation strain tensor $\mathbf{V}^{\mathbf{f}}$ :

$$
\mathbf{V}^{\mathbf{f}}= \pm \Omega\left[\begin{array}{lll}
0 & & \\
& 0 & \\
& & 1
\end{array}\right]+\frac{V^{r}}{3}\left[\begin{array}{lll}
1 & & \\
& 1 & \\
& & 1
\end{array}\right]
$$

The + sign is for vacancy formation and the - sign is for interstitial formation throughout this paper. $\Omega$ is the atomic volume, representing the dimension changes of the crystal upon creation or

from the weighted contributions of those several mechanisms. In the ensuing discussion we assume a single mechanism for simplicity.

$¥$ Neglecting variations in $v$ and $\lambda$ is practically always justified $[19,20]$, but the pressure-dependence of $f$ may or may not be negligible, depending on whether there is a significant pressuredependence to the interaction between the impurity and the defect. This pressure effect is analogous to the additional term in the expression for the apparent activation enthalpy introduced by a significantly temperature-dependent binding, which has been analyzed by Hu [21]. 
annihilation of a lattice site, before permitting relaxation of the lattice around the newly-created point defect. The relaxation volume $V^{r}$ propagates out elastically to all of the sample surfaces and therefore provides equal contributions along all axes. The dependence of the equilibrium defect concentration $C^{e}$ on applied stress $\sigma$ can be written

$$
\frac{C^{e}(\boldsymbol{\sigma})}{C^{e}(\mathbf{0})}=\exp \left(\frac{\boldsymbol{\sigma} \cdot \mathbf{V}^{\mathbf{f}}}{k_{\mathrm{B}} T}\right)
$$

where $\mathbf{V}^{\mathbf{f}}$ has the elements 0 and 1 placed appropriately in Eq. (6) for the surface at which the defects equilibrate. These dimension changes and their interactions with nonhydrostatic stresses are indicated schematically for a simple case in Fig. 2.

In general, the defect mobility $M$ in directions parallel and perpendicular to the direction of applied stress differ $[17,18] . D_{33}$, the diffusivity in a direction normal to the (001) surface, depends on $M_{33}$, the mobility in this direction. The effect of stress on $M_{33}$ is characterized by the migration strain tensor, which must have the form

$$
\mathbf{V}_{\mathbf{3 3}}^{\mathbf{m}}=\left[\begin{array}{ccc}
V_{\perp}^{m} & & \\
& V_{\perp}^{m} & \\
& & V_{\|}^{m}
\end{array}\right] .
$$

$V_{\|}^{m}$ and $V_{\perp}^{m}$, respectively, are the dimension changes of the crystal parallel and perpendicular to the direction of net transport when the point defect reaches its saddle point. As the diffusivity is proportional to the product of the concentration and mobility of defects, the effect of stress on the diffusivity in the direction normal to the surface is obtained by the combination of Eqs. (6) and (8):

$$
\begin{aligned}
& \frac{D_{33}(\sigma)}{D_{33}(\mathbf{0})}=\exp \left(\frac{\sigma \cdot \mathbf{V}_{\mathbf{3 3}}^{*}}{k_{\mathrm{B}} T}\right) ; \\
& \mathbf{V}_{\mathbf{3 3}}^{*}=\mathbf{V}^{\mathbf{f}_{+}} \mathbf{\mathbf { V } _ { \mathbf { 3 3 } }} .
\end{aligned}
$$

Hydrostatic pressure then influences $D_{33}$ according to

$$
\frac{D_{33}(p)}{D_{33}(0)}=\exp \left(\frac{-p\left[ \pm \Omega+V^{r}+V^{m}\right]}{k_{\mathrm{B}} T}\right)
$$


where the sum of the bracketed terms in Eq. (11) is the conventionally-defined scalar activation volume, and the conventionally-defined scalar migration volume $V^{m}$ is the trace of Eq. (8). Biaxial stress influences $D_{33}$ according to

$$
\frac{D_{33}\left(\sigma_{\text {biax }}\right)}{D_{33}(0)}=\exp \left(\frac{\sigma_{\text {biax }}\left[\frac{2}{3} V^{r}+V^{m}-V_{\|}^{m}\right]}{k_{\mathrm{B}} T}\right) .
$$

\section{EXPERIMENTAL RESULTS}

There are very few measurements of the activation volume for diffusion in $\mathrm{Si}$. This is largely because such an experiment requires a pressure medium that neither reacts with, contaminates, nor plastically deforms silicon at high temperature. In early experiments we found negative activation volumes for $\mathrm{As}$ [22] and $\mathrm{Si}$ [23] diffusion using a $\mathrm{NaCl}$ pressure medium. However, the $\mathrm{NaCl}$ pressure medium introduces substantial uncertainties into the results, especially for the self-diffusion study. Because Si is such a slow diffuser, this experiment had to be performed at $T$ above the $p=0$ melting point of $\mathrm{NaCl}$, requiring that the $p=0$ anneals be performed in a different furnace than the high- $p$ anneals and introducing the possibility of temperature cross-calibration artifacts. Until the results are reproduced in "cleaner" experiments, we cannot have much confidence in them. More recently, activation volumes were measured for B and Ga diffusion in Si by Södervall et al. [24] using a gas apparatus. However, as the initial profiles were ion implanted, an undetermined amount of transient enhanced diffusion may be affecting the results.

Very recently we have used a clean and hydrostatic liquid Ar pressure medium and measured a pressure-enhanced diffusivity of B [12] in $\mathrm{Si}$, characterized by an activation volume of $V^{*}=-0.17 \Omega$, and a pressure-retarded diffusivity of $\mathrm{Sb}[13]$ in $\mathrm{Si}$, characterized by an activation volume of $V^{*}=$ $+0.07 \Omega$. Note that these values are much smaller in magnitude than one atomic volume, and that such a small pressure effect implies from (11) that $V^{r}+V^{m}$ is large enough in magnitude to nearly offset the $\pm \Omega$ term. Hence we should expect the stress effect in (12) to be relatively large.

The influence of biaxial stress on the diffusivity has been characterized experimentally [2-8] by the apparent change in activation energy with biaxial (tensile) strain, $\varepsilon_{\text {biax }}$, at constant composition: 


$$
Q^{\prime} \equiv-k_{\mathrm{B}} T \frac{\partial \ln D_{33}}{\partial \varepsilon_{\mathrm{biax}}}
$$

By comparison to Eq. (12), the combination of volumes in square brackets in Eq. (12) is equal to $-Q^{\prime} / Y$, where the biaxial modulus $Y$ is the ratio of Young's modulus to one minus Poisson's ratio. Additionally, one must take care to isolate experimentally the stress effect from the composition effect; this has been done in some of the most recent experiments $[5,7,8]$.

To predict the hydrostatic or biaxial stress effects with Eqs. (11) or (12), respectively, the three parameters $V^{r}, V_{\|}^{m}$, and $V_{\perp}^{m}$ must be known. However, certain combinations of experimental observables are independent of some of the parameter values. Combining Eqs. (8), (11), (12) and (13),

$$
V^{*}+\frac{3}{2} \frac{Q^{\prime}}{Y}= \pm \Omega+\left(V_{\|}^{m}-V_{\perp}^{m}\right)
$$

If we assume that the anisotropy in the migration strain tensor is negligible, then the right-hand side of Eq. (14) should be $+1 \Omega$ for a vacancy mechanism and $-1 \Omega$ for an interstitial-based mechanism.

For the simple vacancy mechanism, or any mechanism whereby point defect jumps are in the $<111>$ directions, the anisotropy in the macroscopically-measured migration volume in $\{001\}$ films should be zero because crystal symmetry dictates that the effect of, say, compression in the [001] and [100] directions on the rate of vacancy jumping in the [111] direction must be identical [10]. (This is not necessarily the case for the interstitialcy or kick-out mechanism, in which the migration path may involve second-neighbor jumps in $\langle 110\rangle$ directions [25], but it would be the case for a migration path that takes an interstitial from one tetrahedral site to an adjacent one via a hexagonal saddle-point.)

The cases of $\mathrm{Sb}$ and B diffusion in $\mathrm{Si}$ are particularly simple because there is a growing consensus that the former diffuses almost entirely by a vacancy mechanism and the latter diffuses almost entirely by an interstitial-based mechanism [26,27]. Eq. (14) with zero migration-strain anisotropy was used [10] to compare the value of $Q^{\prime}$ reported by Kringhoj et al. [8] for $\mathrm{Sb}$ diffusion in biaxially strained $\mathrm{Si}$ and $\mathrm{Si}-\mathrm{Ge}$ and $V^{*}$ measured in hydrostatically compressed $\mathrm{Si}$. The result is $V^{*}+\frac{3}{2} \frac{Q^{\prime}}{Y}=(+0.93 \pm$ 0.20) $\Omega$ for a compressively strained $\mathrm{Si}_{91} \mathrm{Ge}_{9}$ alloy and $V^{*}+\frac{3}{2} \frac{Q^{\prime}}{Y}=(+1.20 \pm 0.33) \Omega$ for tensile $\mathrm{Si}$. 
With no free parameters, these results are in excellent agreement with the prediction of $+1 \Omega$ for a simple vacancy mechanism. Graphically, this agreement is illustrated in Fig. 3, where the biaxial strain effect is predicted from the hydrostatic measurement using Eq. (14) and compared to the measured biaxial effect.

A similar analysis has been performed for the existing experiments on B diffusion in $\mathrm{Si}[7,11,12]$, yielding qualitative agreement assuming a predominantly interstitial-based mechanism as illustrated in Fig. 4. However, in this case the migration strain anisotropy is not necessarily zero and has not yet been measured or determined theoretically by independent means. We can therefore interpret Fig. 4 as a "measurement" of the migration strain anisotropy - a determination of the value of the anisotropy required to reconcile the observed hydrostatic and biaxial experimental results with an interstitialbased mechanism. However, because the biaxial result of Kuo et al. in pure Si displays a different slope than their biaxial results in the Si-Ge alloys, one must be very cautious about over interpreting these results at this time, before a more thorough understanding of the various influences is available. The slope in Fig. 4 predicted from the hydrostatic experiment in unalloyed Si for a migration strain anisotropy of $V_{\|}^{m}-V_{\perp}^{m}=+\Omega / 2$ (Fig. 4(c)) is consistent with the slopes of the data from the biaxial experiments for the alloys but is not consistent with the slope of the biaxial effect for unalloyed $\mathrm{Si}$. A migration strain anisotropy of $V_{\|}^{m}-V_{\perp}^{m}=+1.2 \Omega$, required to reconcile the biaxial and hydrostatic effects in unalloyed $\mathrm{Si}$, is so large in magnitude that it borders on the implausible. Other indirectly related experiments and calculations are also interpreted [12], using various assumptions, and the resulting predictions are plotted; they all display general agreement with the biaxial alloy data but not with the unalloyed Si data. Potential reasons for the negative slope of the unalloyed Si data and the positive slope of everything else are currently under investigation.

\section{PREDICTING ARBITRARY STRESS EFFECTS}

From Eqs. (6) and (8)-(10) it is apparent that for describing diffusion normal to the (001) surface, the three parameters $V^{r}, V_{\|}^{m}$, and $V_{\perp}^{m}$ appear in only two independent combinations, $V^{r} / 3+V_{\perp}^{m}$ and $V^{r} / 3+V_{\|}^{m}$. Hence if there is a single defect mechanism and charge state dominating transport then the 
prediction of the effect of an arbitrary stress state on $D_{33}$ requires the theoretical determination of the three aforementioned parameters or only two independent measurements (e.g., biaxial and hydrostatic stress effects); if symmetry requires the migration strain anisotropy to be zero then the number is further reduced. In the fabrication of small devices, diffusion in directions other than normal to the surface becomes important. We therefore discuss what further progress is required to permit the prediction of the effect of an arbitrary stress state on diffusion in an arbitrary direction.

The theoretical framework requires some further development. If there are multiple species and charge states involved in diffusion, clearly the contributions from each must be superposed. Additionally, strain breaks the energetic degeneracy of otherwise symmetry-equivalent ground states as well as saddle configurations; in principle they must all be enumerated and superposed (though under point-defect equilibrium conditions the effect of stress on ground-state energetics cancels out of the expression for the effect of stress on the diffusivity [17]). In effect, the treatment presented above averages over the otherwise symmetry-equivalent configurations, which is expected to be valid if the degeneracy splitting $« k_{\mathrm{B}} T$ but not otherwise. Additionally, in a crystal structure whose primitive cell has a multiple-atom basis such as $\mathrm{Si}$, each site in the basis set must be tracked separately [29]. (This complication is not necessary for diffusion by a vacancy mechanism normal to the (001) surface, due to the symmetry of the sites with respect to this surface.)

In general the activation strain tensor has 81 elements, none of which is necessarily zero:

$$
D_{\mathrm{ij}}(\sigma)-D_{\mathrm{ij}}(0) \sim V_{\mathrm{ijkl}}^{*} \sigma_{\mathrm{kl}},
$$

where the sum over repeated indices is implied. Fortunately, often symmetry will dictate that only a handful be independent. For a given mechanism (ground state - saddle combination) all of the information about the macroscopic activation strain tensor and its 81 elements is contained within the local configuration change during the transition from the ground state to the saddle configuration. For this transition, the migration strain tensor reckoned from a coordinate system with one axis (say, the third) along the jump direction [29] is given by 


$$
\Omega_{\text {local }}^{\mathbf{m}}=\left[\begin{array}{ccc}
\Omega_{\perp 1}^{\mathrm{m}} & \Omega_{12}^{\mathrm{m}} & \Omega_{13}^{\mathrm{m}} \\
\Omega_{12}^{\mathrm{m}} & \Omega_{\perp 2}^{\mathrm{m}} & \Omega_{23}^{\mathrm{m}} \\
\Omega_{13}^{\mathrm{m}} & \Omega_{23}^{\mathrm{m}} & \Omega_{\|}^{\mathrm{m}}
\end{array}\right]
$$

which has at most six independent elements. Transforming to the crystallographic coordinate system and superposing the contributions for the various defect ground-state and saddle-point orientations should then lead to all 81 elements. If the saddle point configuration is sufficiently symmetric the offdiagonal elements of $\Omega_{\text {local }}^{\mathbf{m}}$ are all zero and the two perpendicular components will be equal to each other, reducing the number of independent elements to two. Note, however, that even in a structure as symmetric as $\mathrm{Si}$, the situation is more complex than this. The isolated vacancy is believed to have tetragonal Jahn-Teller distortions [30]. The diffusion of donor impurities by a vacancy mechanism is believed to not have a saddle-point configuration in which the impurity has jumped half way into the vacancy, but rather to have one in which the impurity and vacancy are at or near third-neighbor sites at opposite ends of a hexagonal ring [21,31,32], in which case the off-diagonal elements of $\Omega_{\text {local }}^{\mathbf{m}}$ are not zero. Some of the outstanding issues discussed above are being addressed in current research [29], which nevertheless still needs to account for the non-local character of the formation strain tensor (Fig. 2) when point defects equilibrate with a nearby surface.

Once the thermodynamic formalism is complete, the determination of the handful of elements in the formation strain tensor and in $\Omega_{\mathbf{l o c a l}}^{\mathbf{m}}$ will permit the prediction of the effect of an arbitrary stress state on diffusion in an arbitrary direction. These parameters can be determined by $a b$ initio density functional theory if all of the atoms within the supercell are permitted to relax and the supercell is permitted to undergo arbitrary strains. Certain combinations of these parameters are also observable macroscopically by designing experiments that isolate the effect of certain stress states on diffusion in certain directions. This combination of theory and experiment is a promising area for the testing of theoretical Hamiltonians and methodologies and of models of the relevant diffusion mechanisms. Agreement should be expected so long as the point defect populations remain in equilibrium with the free surface or with some other well-known sink. During transient, non-equilibrium processes such 
as defect reactions and clustering, strain tensors associated with all the kinetic saddle points and metastable species may also be important.

\section{SUMMARY}

Point defect mechanisms are related through thermodynamics to the dependence of the diffusivity on pressure and stress. The influences of hydrostatic and biaxial stress are interdependent in a predictable way for any proposed mechanism. For $\mathrm{Sb}$ in $\mathrm{Si}$, the measured effect of biaxial stress on diffusion can be predicted successfully from the hydrostatic results with no free parameters. For B in $\mathrm{Si}$, the migration strain anisotropy can be treated as a free parameter and adjusted to reconcile hydrostatic and biaxial results; however an implausibly large value is needed in the case of unalloyed Si. Finally, it appears that the means to predict the effect of an arbitrary stress state on diffusion in an arbitrary direction are within reach.

\section{ACKNOWLEDGMENTS}

This research was supported by NSF DMR-98-13803. I am grateful to my collaborators Y. Zhao, H.-J. Gossmann, S. Mitha and D. Schiferl in the experimental work reviewed here and to M.S. Daw for helpful discussions and for the communication of his theoretical results prior to publication. 


\section{REFERENCES}

${ }^{1}$ P.M. Fahey, P.B. Griffin and J.D. Plummer, Rev. Mod. Phys. 61, 289 (1989).

${ }^{2}$ N. Moriya, L.C. Feldman, H.S. Luftman, C.A. King, J. Bevk and B. Freer, Phys. Rev. Lett. 71, 883 (1993).

${ }^{3}$ F.H. Baumann, J.-H. Huang, J.A. Rentschler, T.Y. Chang and A. Ourmazd, Phys. Rev. Lett. 73, 448 (1994).

${ }^{4}$ N.E.B. Cowern, P.C. Zalm, P. van der Sluis, D.J. Gravesteijn and W.B. de Boer, Phys. Rev. Lett. 72, 2585 (1994).

${ }^{5}$ N.E.B. Cowern, W.J. Kersten, R.C.M. de Kruif, J.G.M. van Berkum, W.B. de Boer, D.J. Gravesteijn and C.W.T. Bulle-Liewma, in Proc. 4th Int. Symp. on Process Physics and Modeling in Semiconductor Devices; eds. G.R. Srinivasan, C.S. Murthy, and S.T. Dunhan, Electrochem. Soc. Proc. 96-4 (Electrochemical Society, Pennington NJ, 1996).

${ }^{6}$ P. Kuo, J.L. Hoyt, J.F. Gibbons, J.E. Turner, R.D. Jacowitz and T.I. Kamins, Appl. Phys. Lett. 62, 612 (1993).

${ }^{7}$ P. Kuo, J.L. Hoyt, J.F. Gibbons, J.E. Turner and D. Lefforge, Appl. Phys. Lett. 66, 580 (1995).

${ }^{8}$ P. Kringhoj, A. Nylandsted-Larsen and S.Y. Shirayev, Phys. Rev. Lett. 76, 3372 (1996).

${ }^{9}$ H. Park, K.S. Jones, J.A. Slinkman and M.E. Law, J. Appl. Phys. 78, 3664 (1995).

${ }^{10}$ M.J. Aziz, Appl. Phys. Lett. 70, 2810 (1997).

${ }^{11}$ M.J. Aziz, Mater. Res. Soc. Symp. Proc. 469, 37 (1997).

${ }^{12}$ Y. Zhao, M.J. Aziz, H.J. Gossmann, S. Mitha and D. Schiferl, Appl. Phys. Lett. 74, 31 (1999).

${ }^{13}$ Y. Zhao, M.J. Aziz, H.J. Gossmann, S. Mitha and D. Schiferl, Appl. Phys. Lett. 75, 941 (1999).

${ }^{14}$ G.H. Vineyard, J. Phys. Chem. Solids 3, 121 (1957).

${ }^{15}$ F.C. Larche and J.W. Cahn, Acta Metall. 33, 331 (1985).

${ }^{16}$ B.J. Spencer, P.W. Voorhees and S.H. Davis, J. Appl. Phys. 73, 4955 (1993).

${ }^{17}$ P.H. Dederichs and K. Schroeder, Phys. Rev. B 17, 2524 (1978).

${ }^{18}$ M.J. Aziz, P.C. Sabin and G.-Q. Lu, Phys. Rev. B 44, 9812 (1991).

${ }^{19}$ R.N. Jeffery and D. Lazarus, J. Appl. Phys. 41, 3186 (1970). 
${ }^{20}$ M. Werner, H. Mehrer and H.D. Hochheimer, Phys. Rev. B 31, 3930 (1985).

${ }^{21}$ S.M. Hu, Phys. Stat. Sol. (b) 60, 595 (1973).

${ }^{22}$ E. Nygren, M.J. Aziz, D. Turnbull, J.M. Poate, D.C. Jacobson and R. Hull, Appl. Phys. Lett. 47, 105 (1985).

${ }^{23}$ M.J. Aziz, E. Nygren, W.H. Christie, C.W. White and D. Turnbull, Mater. Res. Soc. Symp. Proc. 36, 101 (1985).

${ }^{24}$ U. Sodervall, M. Friesel and A. Lodding, J. Chem. Soc. Faraday Trans. 86, 1293 (1990).

${ }^{25}$ M. Tang, L. Colombo, J. Zhu and T. Diaz de la Rubia, Phys. Rev. B 55, 14279 (1997).

${ }^{26}$ H.-J. Gossmann, T.E. Haynes, P.A. Stolk, D.C. Jacobson, G.H. Gilmer, J.M. Poate, H.S. Luftman, T.K. Mogi and M.O. Thompson, Appl. Phys. Lett. 71, 3862 (1997).

${ }^{27}$ A. Ural, P.B. Griffin and J.D. Plummer, J. Appl. Phys. 85, 6440 (1999).

${ }^{28}$ A. Antonelli and J. Bernholc, Phys. Rev. B 40, 10643 (1989).

${ }^{29}$ M.S. Daw, W. Windl, N.N. Carlson, M. Laudon, and M.P. Masquelier, Phys. Rev. B, to be published.

${ }^{30}$ A. Antonelli, E. Kaxiras and D.J. Chadi, Phys. Rev. Lett. 81, 2088 (1998).

${ }^{31}$ O. Sugino and A. Oshiyama, Phys. Rev. B 46, 12335 (1992).

${ }^{32}$ J. Xie and S.P. Chen, J. Phys. D 32, 1252 (1999).

a)maziz@ harvard.edu; http://www.deas.harvard.edu/matsci 


\section{FIGURE CAPTIONS}

FIG. 1. Schematic of volume changes associated with diffusion by vacancy (top) and interstitialcy (bottom) mechanism. Pressure effect is determined by volume changes of sample upon defect formation $\left(V^{f}\right)$ and migration $\left(V^{m}\right)$, which are shown. To create a vacancy, one moves an atom from the interior to a surface site; to create an interstitial, one moves an atom from a surface site to the interior. Stress effect is determined by shape change of sample upon defect formation and migration and, for formation, depends upon which surface the defect is formed from. (If point defect equilibration occurs at an internal dislocation instead of the surface, dislocation climb results in an identical volume change; however, the shape change depends on the direction of the Burgers vector.) Stress-strain work done by point defect formation and migration is indicated by interactions of shape changes with stresses (thin arrows). For simplicity only first half of interstitialcy mechanism is shown.

FIG. 2. Work done upon vacancy formation in crystal (middle) with stepped surfaces free of extended defects. Pressures, $p_{1}$ and $p_{3}$, of incompressible fluids in contact with (100) and (001) faces, respectively, maintained by pistons driven by masses $m_{1}$ and $m_{3}$. Crystal volume changes by $V f$ upon vacancy formation at (100) or (001) surface, but work done against gravity differs.

FIG. 3. Biaxial strain effect on Sb diffusion in Si-Ge thin film at constant composition. Data points represent average values from Kringhoj et al. over range $900-1030{ }^{\circ} \mathrm{C}$. The slopes of the lines indicate the value of $Q^{\prime}$ expected from the biaxial experiment by inputting our experimental result under hydrostatic conditions into Eq. (14) with no free parameters. The offset between lines is a free parameter representing the effect of composition at constant strain; lines have been chosen to go through the data points at zero strain. 
FIG. 4. Comparison of interstitial-based mechanisms in biaxially strained Si-Ge films for the indicated assumed values of migration strain anisotropy. Vertical offsets represent composition effect at constant strain; slopes represent strain effect at constant composition. Data points from Kuo et al. for B diffusion in 0, 10, and 20\% Ge alloys. Curve (a): $Q^{\prime}$ reported for oxidation-enhanced interdiffusion of Si/Ge multilayers by Cowern et al. [5]. Curve (b): B diffusion in Si from Eq. (14) and our measurement of $V^{*}$. Also shown is the strain dependence of the I-based contribution to Si self diffusion, from Eq. (1) using: (c) the TBA calculation of Tang et al. [25] for the <110> dumbbell self-interstitial ground state and assuming $V_{I}^{m}=0$; (d) the ab initio calculation of Antonelli and Bernholc [28] assuming a tetrahedral interstitial saddle point. Curve (e): ratio of $D_{\mathrm{B}}$ parallel $\left(D_{11}\right)$ and perpendicular $\left(D_{33}\right)$ to surface in $\mathrm{Si}(001)$, to show magnitude of diffusion anisotropy expected from various values of migration strain anisotropy. Straight lines chosen to go through unstrained pure Si data point; only slopes are significant except for curve (b). 
VACANCY

INTERSTITIALCY

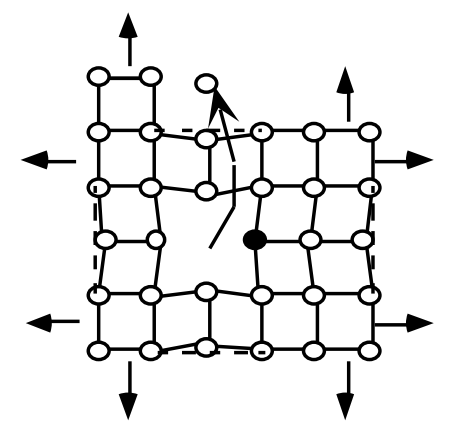

FORMATION

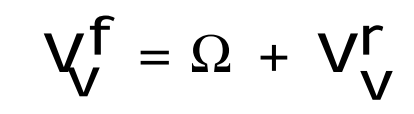

MIGRATION

$v \mathbf{v}_{\mathbf{v}}$

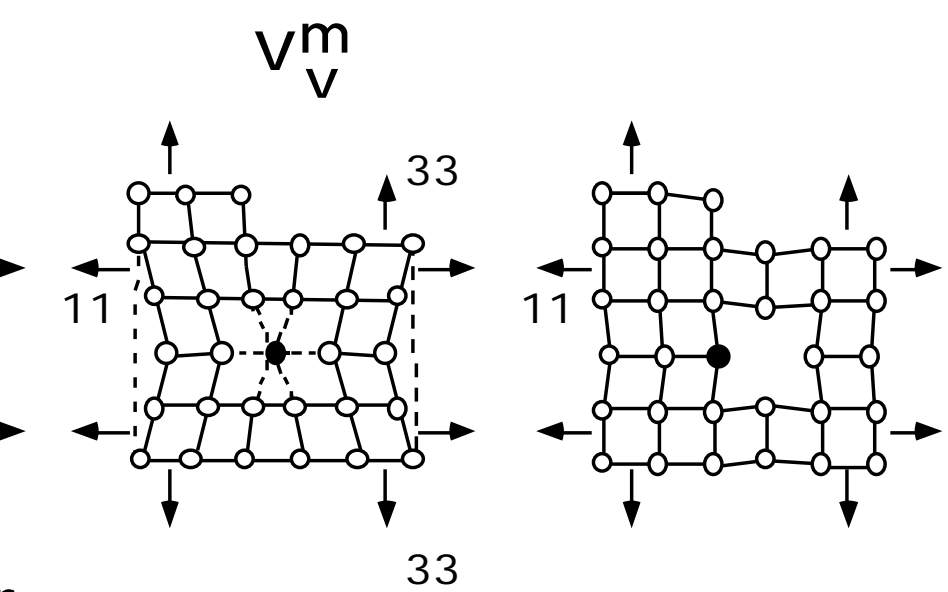

$\mathbf{v} \mathbf{m}$

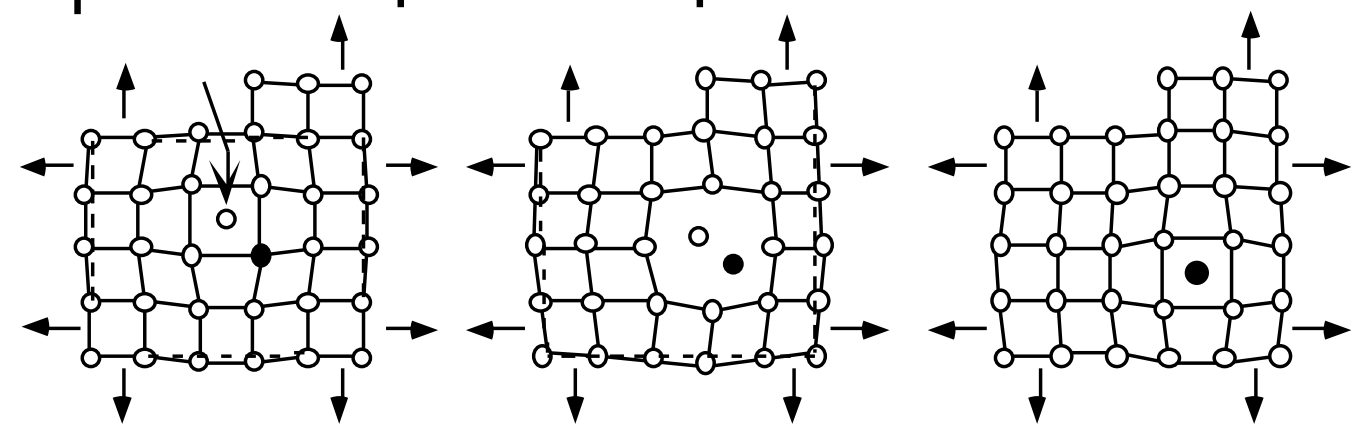

FIG. 1. Schematic of volume changes associated with diffusion by vacancy (top) and interstitialcy (bottom) mechanism. Pressure effect is determined by volume changes of sample upon defect formation $\left(V^{f}\right)$ and migration $\left(V^{m}\right)$, which are shown. To create a vacancy, one moves an atom from the interior to a surface site; to create an interstitial, one moves an atom from a surface site to the interior. Stress effect is determined by shape change of sample upon defect formation and migration and, for formation, depends upon which surface the defect is formed from. (If point defect equilibration occurs at an internal dislocation instead of the surface, dislocation climb results in an identical volume change; however, the shape change depends on the direction of the Burgers vector.) Stress-strain work done by point defect formation and migration is indicated by interactions of shape changes with stresses (thin arrows). For simplicity only first half of interstitialcy mechanism is shown. 


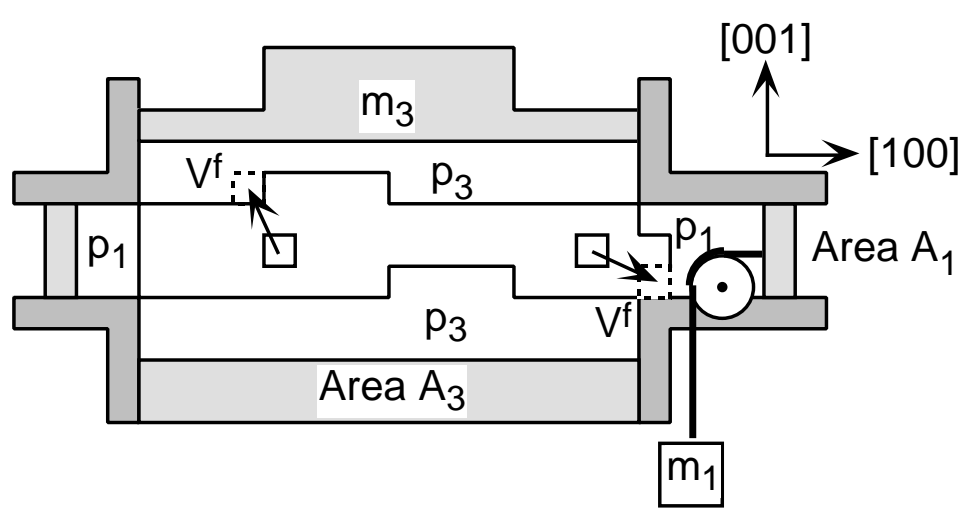

FIG. 2. Work done upon vacancy formation in crystal (middle) with stepped surfaces free of extended defects. Pressures, $p_{1}$ and $p_{3}$, of incompressible fluids in contact with (100) and (001) faces, respectively, maintained by pistons driven by masses $\mathrm{m}_{1}$ and $\mathrm{m}_{3}$. Crystal volume changes by $V f$ upon vacancy formation at (100) or (001) surface, but work done against gravity differs. 


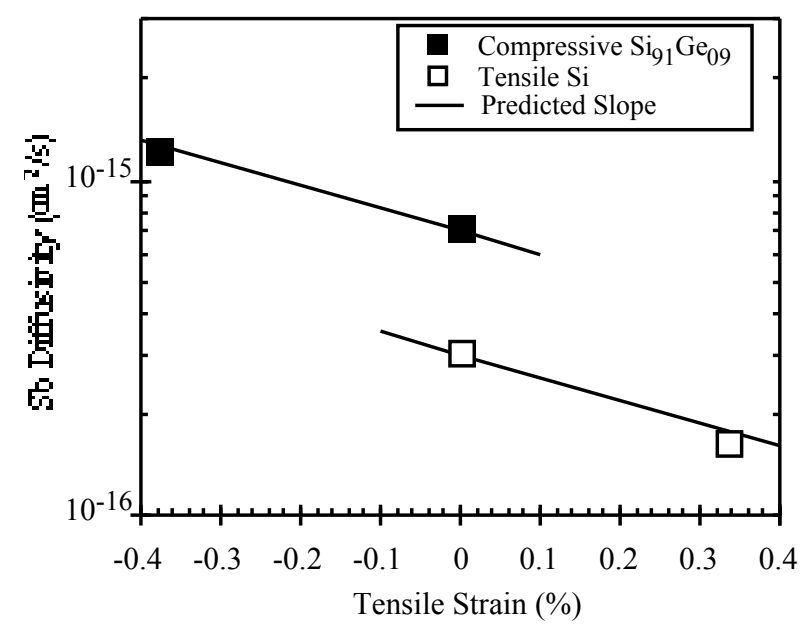

FIG. 3. Biaxial strain effect on Sb diffusion in Si-Ge thin film at constant composition. Data points represent average values from Kringhoj et al. over range $900-1030{ }^{\circ} \mathrm{C}$. The slopes of the lines indicate the value of $Q^{\prime}$ expected from the biaxial experiment by inputting our experimental result under hydrostatic conditions into Eq. (14) with no free parameters. The offset between lines is a free parameter representing the effect of composition at constant strain; lines have been chosen to go through the data points at zero strain. 


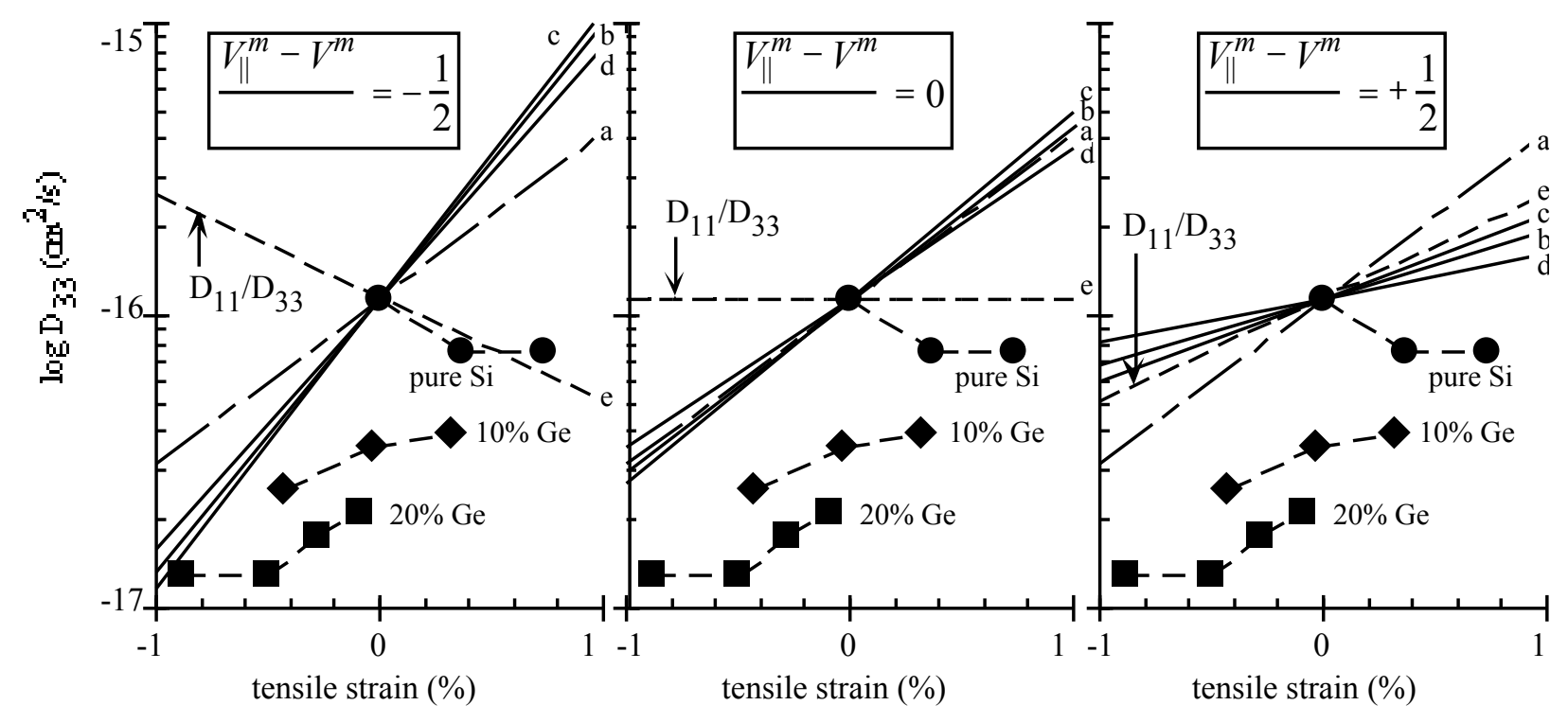

FIG. 4. Comparison of interstitial-based mechanisms in biaxially strained Si-Ge films for the indicated assumed values of migration strain anisotropy. Vertical offsets represent composition effect at constant strain; slopes represent strain effect at constant composition. Data points from Kuo et al. for B diffusion in 0, 10, and 20\% Ge alloys. Curve (a): $Q^{\prime}$ reported for oxidation-enhanced interdiffusion of Si/Ge multilayers by Cowern et al. [5]. Curve (b): B diffusion in Si from Eq. (14) and our measurement of $V^{*}$. Also shown is the strain dependence of the I-based contribution to $\mathrm{Si}$ self diffusion, from Eq. (1) using: (c) the TBA calculation of Tang et al. [25] for the <110> dumbbell self-interstitial ground state and assuming $V_{I}^{m}=0$; (d) the $a b$ initio calculation of Antonelli and Bernholc [28] assuming a tetrahedral interstitial saddle point. Curve (e): ratio of $D_{\mathrm{B}}$ parallel $\left(D_{11}\right)$ and perpendicular $\left(D_{33}\right)$ to surface in $\mathrm{Si}(001)$, to show magnitude of diffusion anisotropy expected from various values of migration strain anisotropy. Straight lines chosen to go through unstrained pure Si data point; only slopes are significant except for curve (b). 